\title{
Routing Performance and Continuous Session Reliability
}

\author{
Stefano-Niko Orzen, Levente Kovács
}

Óbuda University, Bécsi út 96/b, H-1034 Budapest, Hungary

stefano-niko.orzen@phd.uni-obuda.hu, kovacs.levente@nik.uni-obuda.hu

\begin{abstract}
This paper presents the research we have performed on networking technology as a review and summary of our doctoral study. Our work's use is to define real-time communications that are transmitted across the Internet, and we have developed a highresolution work concerning the logic that underlies the routing phenomenon. Routing features were analyzed with respect to current trends and demands that imply the usefulness of resources. Much research exists and is constantly being developed for routing technology at fine grain or grosser scale. As the world needs more and more a means to make way and find paths through the millions of interconnected devices, we have proposed a fault-tolerance methodology that implies reasoning, as well as a concise requirement engineered approach for ensuring QoS. In quality of service, there is the discrepancy of knowing that resources are available and are constantly kept as reserved while different autonomous systems suffer from inadequate resource pooling as a whole. Measurement units such as bits/second, erlangs, and other quantifiable data units have been enabled for the measurement of given networking ability. These abilities are properties of infrastructures or network segments in being capable to send data and all of them fail in front of the dynamic nature of the Internet. As it will be presented, our approach shows that all these units can be more understandably put to use if Queueing Theory, Markov Chains, statistical evaluation, and inter-momentary discrete events are analyzed for the exact context or case that requires an optimal routing decision.
\end{abstract}

Keywords: real-time; communications; infrastructure; session; measurement

\section{Introductory Notes}

The scientific world of computer networks has managed to enable and met a multitude of criteria for requirements that underlie various aspects of how realtime processes work [1]. However, when it comes to reliable communications with continuous availability and fault tolerance, there is a significant shortage of methods that should ensure the correct functioning of related processes. 
In this study, the possibility of tolerating intermediate failures that may occur in persistent route transmissions was developed while mainly focused on what can be achieved by adjusting equipment's performance parameters that enable real-time processes.

Routing equipment holds a central role in information transmissions, and it imposes the use of minimum and maximum limits that shape the communicated data volume through the information media.

The simplest way to represent data communications is provided by the ratio between the transmitted bits per time-frame, and the given value stands as basis for the entire development in data communications.

Real-time communication is a separate field that depends on much more sophisticated and demanding criteria than regular or average Internet and network transmissions [2]. All this is due to the fact that the mechanisms of the protocols considered common have no time limits for transmitting data, and all research in their direction has been aimed at providing performance that does not disturb users of network services. However, in real-time, things are much more complex because the data transmitted between two entities in real-time must reach their destination within a time limit set priorly by the protocol that connects the requesters of such a process.

Persistent routing is defined as a problem because it is a network phenomenon that implies a directly generated process that can be affected by overloaded networks that have the timely delivery possibilities reduced [3].

Bandwidth does not impose difficult problems in the world of data transmission. Factors that lead network segments's overload are critical issues where network availability, robustness and reliability are assigned as properties [4].

Due to the vast number of elements that form real-time communications, the functional chaining of various stages and thresholds (from one level of OSI communication to another) requires the association of indices and parameters to help formalize the analysis of communication performance. In [5] these metrics are defined by their importance in streamlining data packet transmissions, having a pivotal role in optimizing communication processes. In the following table, we present the in-cause metrics together with their brief description, these being a basis for the developed solutions that are claimed in this article.

Table 1

Performance metrics

\begin{tabular}{|c|l|}
\hline Metric & \multicolumn{1}{c|}{ Definition } \\
\hline $\mathrm{C}$ & Servers capacity to take over tasks \\
\hline$\lambda$ & Arrival rate of workloads to servers for processing \\
\hline $\mathrm{r}$ & Waiting time for tasks are in processing centers \\
\hline $\mathrm{R}$ & Response time for tasks that are being processed \\
\hline
\end{tabular}




\begin{tabular}{|c|l|}
\hline $\mathrm{T}$ & Time to complete the processing of tasks that reside in processing centers \\
\hline $\mathrm{U}$ & $\begin{array}{l}\text { Utilization degree of processing centers in relation to the arrival rate of } \\
\text { workloads }\end{array}$ \\
\hline $\mathrm{S}$ & Processing time of the computing station \\
\hline $\mathrm{X}$ & $\begin{array}{l}\text { The volume of tasks processed in the entire point-to-point communication } \\
\text { process }\end{array}$ \\
\hline $\mathrm{D}$ & Volume of tasks/loads that are dropped due to network anomalies \\
\hline
\end{tabular}

Optimizing real-time communication requires a concise knowledge of the various causes and problems that can occur in the interconnection of large data networks, and for the processes of real-time sessions, numerous research and studies have been undertaken such as [6], [7], [8] and [9].

Generally, researchers have presented real-time transmissions as functional, but current studies have shown a viable way to assign routing possibilities and choices for trust and fault tolerance. Most proposed solutions are slight improvements of existing technologies, these being presented in the state-of-the-art research stage of this thesis.

Since the contributions that are claimed in this study have the role of completing the field of computer networks, we will present in the following paragraphs the level at which the original contribution is suitable to the real-time transmissions organized by the OSI model (Table 2) ruleset.

The OSI model is the basic theoretical formulation of any communication network, which is defined correctly and is also conformant to ISO standards. For defined networks that use the OSI model rules, there are some specified criteria that need to be met, all this being imposed to achieve the goal set by the creators of the infrastructure in question.

The most intense researched criteria are fault tolerance and reliability, properties derived from the availability of resources, the robustness of programs that form communication protocols, and the integrity of the information communicated through hybrid environments [10].

In Table 2, a comparison between the classic model of open systems interconnection and the OSI model that is complemented with a proposed Interaction layer is presented. The Interaction Layer is considered an improvement because the logic of the original OSI model needs a more thorough grasp of the newly developed technologies' requirements.

In essence, this additional layer is a methodology through which we propose the analysis of the interactions between equipment and network protocols that act as an autonomous assembly with the role of achieving certain performances. The signals and the network equipment forwarded traffic lead to a global network and system functionalities. The numerous actions and reactions of the existing assemblies from interconnected global structures are dependent on the performances of existing devices and environments. 
Table 2

Interaction layer integration in the OSI model

\begin{tabular}{|c|c|}
\hline $\begin{array}{l}\text { The Classic Open } \\
\text { Interconnection Model }\end{array}$ & $\begin{array}{l}\text { Complete Open Systems Interconnection } \\
\text { Model }\end{array}$ \\
\hline $\begin{array}{l}\text { Notes on the operation of each level in the } \\
\text { model }\end{array}$ & $\begin{array}{l}\text { Mentions about the benefits of completing } \\
\text { the model }\end{array}$ \\
\hline Application & Application \\
\hline $\begin{array}{l}\text { It provides high-level access mechanisms } \\
\text { and protocols for users, ensuring } \\
\text { applications' operation through networks } \\
\text { based on Internet Protocol addresses. }\end{array}$ & $\begin{array}{l}\text { It allows the abstraction of resource } \\
\text { requests, which are transparent for } \\
\text { computing technology users. }\end{array}$ \\
\hline Presentation & Presentation \\
\hline $\begin{array}{l}\text { Implemented to facilitate the process of data } \\
\text { transmission between computing systems } \\
\text { that use different data representation } \\
\text { methods. }\end{array}$ & $\begin{array}{l}\text { It is possible to communicate using various } \\
\text { data representations in a computational } \\
\text { platform that understands the } \\
\text { communication protocol's application. }\end{array}$ \\
\hline Session & Session \\
\hline $\begin{array}{l}\text { Create administration possibilities and a } \\
\text { semi-dialogue between remote points in the } \\
\text { network, ensuring a functional software } \\
\text { system design. }\end{array}$ & $\begin{array}{l}\text { The protocol's analysis through the } \\
\text { commands that facilitate its diagnosis is } \\
\text { supported. }\end{array}$ \\
\hline 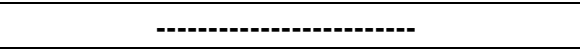 & INTERACTION \\
\hline & $\begin{array}{l}\text { It requires an analysis and diagnosis of the } \\
\text { technical state of operation of a network and } \\
\text { completes the other logical levels through } \\
\text { methods and mechanisms of control over } \\
\text { dependencies, these being critical aspects in } \\
\text { ensuring optimal operation on the correct } \\
\text { use of network resources. }\end{array}$ \\
\hline Transport & Transport \\
\hline $\begin{array}{l}\text { Fragments the data and ensures its } \\
\text { transmission. At this level there are also } \\
\text { protocols for directing and organizing } \\
\text { traffic from very wide environments, with a } \\
\text { very high data flow and degree of } \\
\text { connectivity. }\end{array}$ & $\begin{array}{l}\text { Implements the rules of the proposed } \\
\text { communication protocol for data } \\
\text { transmission over extended networks. }\end{array}$ \\
\hline Network & Network \\
\hline $\begin{array}{l}\text { Assigns network addresses and also ensures } \\
\text { higher levels of the correct configuration of } \\
\text { Levelsor } 1 \text { and } 2 \text {. }\end{array}$ & $\begin{array}{l}\text { Ensures the allocation of arguments } \\
\text { depending on the active network identifiers } \\
\text { and how to manage those identifiers in the } \\
\text { software platform created by the proposed } \\
\text { protocol. }\end{array}$ \\
\hline Data Link & Data Link \\
\hline $\begin{array}{l}\text { Ensures the correctness of the physical } \\
\text { implementation through an electronic } \\
\text { connection between the equipment. At this } \\
\text { level, hardware-coded addresses (e.g. MAC, }\end{array}$ & $\begin{array}{l}\text { Supports communication protocol and } \\
\text { software interface with efficient and correct } \\
\text { logical implementation. }\end{array}$ \\
\hline
\end{tabular}




\begin{tabular}{|l|l|}
\hline $\begin{array}{l}\text { IMEI) are registered in the records of the } \\
\text { equipment that will support the other levels } \\
\text { of communication. }\end{array}$ & \\
\hline Physical & Physical \\
\hline $\begin{array}{l}\text { Ensures proper implementation of hardware } \\
\text { and physical connections (e.g. ethernet and / } \\
\text { or fiber optic, wireless, satellite cablinge). }\end{array}$ & $\begin{array}{l}\text { Allocates physical resources for data } \\
\text { transmission and computation with related } \\
\text { technical limitations. }\end{array}$ \\
\hline
\end{tabular}

As an example of the multitude of interactions that can exist in real-time communication processes, we mention the waiting times of data packets in session-level transmissions that terminate a faulty connection. In connectionoriented communications, data packets must be acknowledged by routers that transmit them and by the computers that are the transmitters and receivers of the in-cause packet.

Data packet verification and control algorithms require routers to store data in their memory until they receive acknowledgments of their receival from the points to which they were transmitted. If a router is overloaded and cannot send session packet confirmation messages in a timely manner, the equipment that waits for an acknowledgement can retransmit the in-cause packet/s and wait again for acknowledgements, or it can actually give up on all unconfirmed packets.

\section{State of The Art Technology Correlation and Current Trends}

There are many dysfunctionalities that can disrupt the operation of persistent routes through which session data is transmitted in real-time, these having various methods for acting on real-time processes. In the performed documentation process, we have distinguished a multitude of negative effects that can disrupt these demanding transmissions from a qualitative point of view [11]. In general, the adverse effects result from parameters based on time quantification, especially for equipment that does not fall within the upper and lower limits of synchronization of end-to-end communications [12].

When data packets are not transmitted in optimal intervals, a multitude of blocking actions that affect persistent routes occur [13]. These blocking actions have as a theoretical explanation the incoherent access to the memories of the communication equipment [14], the latter not being able to execute the instructions of the real-time protocols in the necessary time intervals that are set as criteria by the performance metrics. Communication times facilitate the creation of time windows to streamline the flow of information through networks [15] and in real-time sessions, these space propagation windows are the continuous chaining of data packets. 
In the studies undertaken by various authors such as [16], [8], and [17], various notations are used to express the usage of time as a finite interval in the transmission of amounts of information through routing media. Throughout this document, a multitude of notions are presented and each variation or resemblance will be pointed out to emphasize the context in which they were introduced.

The synchronization algorithm we propose as a contribution alongside the OSI interaction layer is applying a temporal evaluation by which the chosen transmission paths are considered the best decisions to continue a real-time process in an active state.

Network equipment synchronization is applied worldwide and generally, UTC standardizations allow a realistic approach to handling time interval indices from data packets. What has not been considered so far in the case of real-time sessions traversed routes is that the fault tolerance of a disturbance from a segment that's performing the transmission can be achieved by transmitting data on a segment capable to take over the initial transmission and which at the same time respects the criteria to transmit the information of the decommissioned segment. To highlight this fact, in the following paragraphs the composition of the designed algorithm is presented in order to establish the continuous transmission performance of persistent routes, a component that operates from the perspective of three main indices that we have defined, namely $\mathrm{T}$, $\mathrm{t}$ and $\mathrm{t}$ '.

An allocated time $\mathrm{T}$ is considered the starting point for data packets from source to destination. In communications between two entities this time $\mathrm{T}$ is unique for both entity 1 and entity 2 because real-time telephony, video, and data transmissions can be from both participants of the communication process at the same time. A time limit allocation $t$ follows, limit in which the actual time to transmit the data from one point to another is framed while the time unit $t^{\prime}$ includes the time required to communicate on alternative routes data that can only be transmitted during a normal operating time limit $t$. These notions are presented in the following listing because they are used in this document to describe various stages of communication performance processes in the application of the contributions and solutions that are proposed:

1) $\mathrm{T}=$ global time for entities participating in the real-time communication process;

2) $t=$ time required to transmit data between the entities participating in the communication process;

3) $t^{\prime}=$ time required to transmit data from the transmission process on an alternate route.

Performances based on time are a part of the characteristics of the developed synchronization algorithm, along with the condition that these need to be satisfied by the devices that will act when failures appear in real-time communications. Routers and switches perform in accordance with the rules of the communication 
protocol by which the data is guided on different routes and together with the technology types, they represent a whole of information that is part of the communication process. The proposed algorithm acts at the logical level of the OSI model and for fault tolerance to happen, we will mention gradually the protocol operation mode in the following listing.

- $\quad$ step 1: the network faulty router or region is identified by the remaining active devices (those found in the vicinity of the defective equipment) of the real-time communication process;

- $\quad$ step 2: the remaining active routers consult the parametric data of the realtime communication process;

- $\quad$ step 3: the parameters necessary for real-time communication for an alternative route are set (time t' defined between two approximate limits);

- step 4: the router lists the neighboring active routers found still in operation, the prior hop to the faulty equipment;

- $\quad$ step 5: the pre-fail node router communicates to the neighboring routers the request to find a path to the subnet or the destination endpoint of the communication process, provided that the route to be selected respects the required performance criteria namely time t';

- $\quad$ step 6: if multiple positive responses from routers are received, the next step proceeds with choosing the router that corresponds most optimally with the criteria of correct communication time usage and most correct operational usage history;

- $\quad$ step 7: the data is communicated to the router, which can provide the necessary time $t^{\prime}$ to be contained in the $\mathrm{T}$ global time necessary for realtime communication between the endpoints of the persistent route;

- step 8: endpoints confirm the receival of data packets and continue transmitting without interrupting the communication process;

Real-time communication protocols of the OSI session layer are considered from the design stages [18] as having a minimum requirement of resources to transmit data. In this manner, $\mathrm{T}$ global time and interval $\mathrm{t}$ can be obtained from the technical specifications of protocols. The problem given by persistent routing (Fig. 1) has time $t^{\prime}$ as a resolution for communication conflicts in routing. This is because that although a segment can be out of order, or even entire networking regions that include classes of IP addresses and subnets that can be decommissioned, there are alternative routes that fall within the limits of $t$ '.

Having time frames measured and incorporated by the transmission process, the averages of transmission units allow the observation of the logical coordination from transmission equipment [19] which is more difficult to achieve due to the complex functional dynamics from computer networks capable of real-time communications. 
The synchronization algorithm and the interaction concept are two solutions that protocol communication rules can implement. Any communication protocol requires optimal algorithmic coordinated agreements that are beneficial to good data flows through interconnected media [20] and for real-time transmissions, the procedure for tolerating faults that we have created is embedded in the protocol logic from Fig. 2.

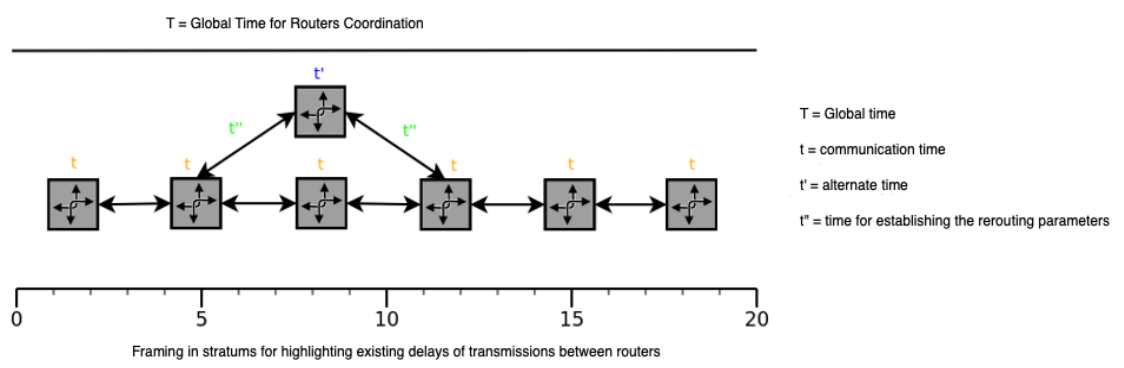

Figure 1

Mapping time requirements

The notions from Fig. 2 are defined to present the essential points for the selection process of an alternative route through which fault tolerance of real-time sessions may occur. These are very closely linked in terms of data packet parameters, and because networks function on a general basis, the problem is given by the possibility of the networking environment to sustain multiple transmissions with a high degree of qualitative criteria.

The TTLs, TCP sequences, time windows, and checksums are represented in the diagram from Fig. 2 in the form of a relation $X=N / R$, where the indices are logical correspondents to the operation of the computational assembly. The assembly functions as analysis through which performance is parametrically adjusted at the equipment level, the latter being a component, part of a multidevice cooperation that's guided by the real-time communication protocol of the session layer. This was mentioned because not all sessions have a real-time runtime. Depending on the type of application (eg. broadcasts, multicast and control terminals), various mechanisms such as character type devices [3] and those of type intermediate memory block are allocated for protocols that can trigger the data packets of communication sessions with a high waiting time.

Alternative routes are selected to continue an affected transmission on certain network segments. This is the main validation method of the proposed solution. As communication routes exist as being available in various intermediate networks, through these routes data packets can be transmitted by the persistent routing protocol. To be taken into account is that the alternative route to be chosen (the one that complies with the allocation criteria t') is to promote the immediate, continuous operation of the transmission process. 


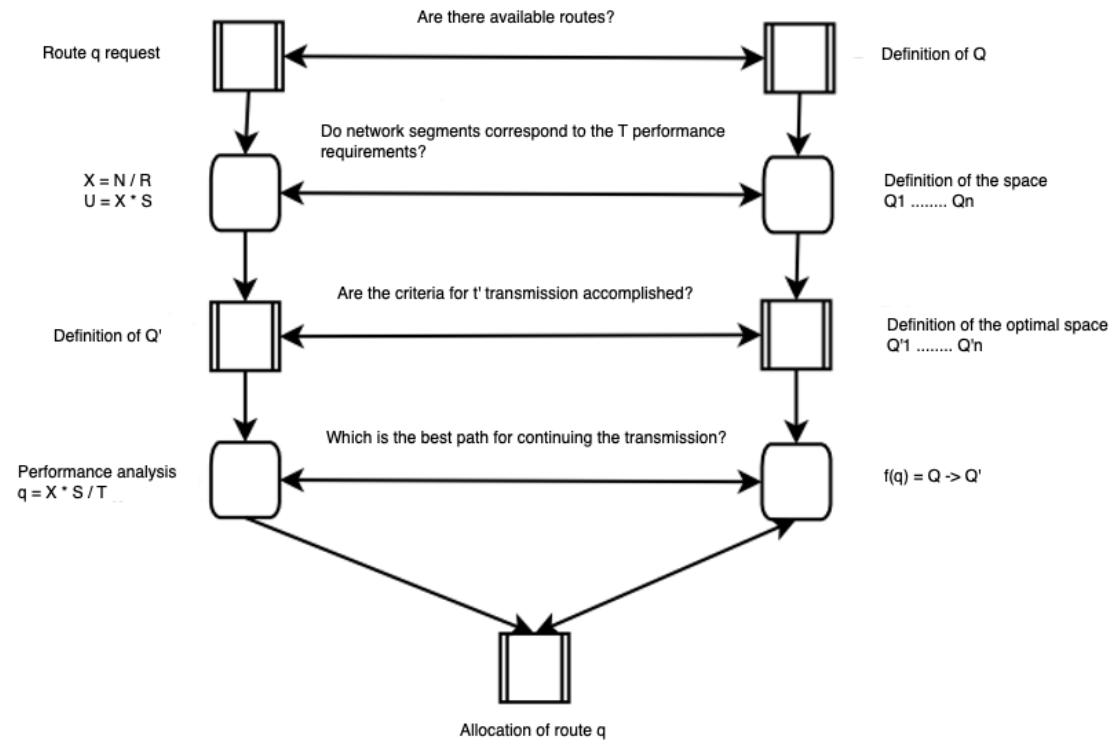

Figure 2

Protocol logic with functional dependencies

Routers have a multitude of ways to allocate alternative segments, but in the process of allocating a communication that will include $t$ ', this allocation is made following an autonomous decision taken by the network based on the parameters of the communication protocol. Thus, the entire process of allocating alternate segments will include the number of existing segments of the router that's adjacent to the affected one, plus the first neighbors of that router.

The definition of the selection range is given by the fact that the topologies, functions, and protocols for renewing routing tables are implemented to make the choice of shortest paths from extended networks, a mode of operation without which Internet traffic could not run [14].

Continuing is the presentation of the selection process for route ranges that can be considered alternative segments. When renewing routing tables, data traffic management equipment provides a new index with all the existing segments that are active. This index contains the number of routes that can be selected to forward packets to other devices, routes that can include service types, and continuous streams. The results presented in this section represent the synchronization algorithm applied on the simulated routing contexts, contexts in which the obtained performances indicate a degree of reliability that can be assigned to the algorithm. 
The measurement metrics for the waiting times in networks stand as a base for the developed synchronization algorithm. The algorithm is demonstrated with its usefulness based on the defining components of any persistent routing process, namely the network search space and the criteria for allocating a route for transmission.

In the following notations, we present the identification of the two components necessary for the operation of the process. They are listed because it is necessary to understand the choice of the alternate forwarding route from a multitude of available alternative routes.

- $\mathrm{Q}=$ available route;

- $\quad\left(\mathrm{Q}_{1} \ldots \mathrm{Q}_{\mathrm{n}}\right)=$ the selection space composed of the available routes that can offer the choice of an alternative route that has the corresponding requirements;

- $\quad\left(\mathrm{Q}^{\prime}{ }_{1} \ldots . . \mathrm{Q}_{\mathrm{n}}^{\prime}\right)=$ limited space to allocate a resource after performing the appropriate requirements analysis;

- $\quad \mathrm{Q}^{\prime}=$ restricted space for the allocation of a communication route;

- $\quad \mathrm{q}=$ chosen route;

- $\quad \mathrm{f}(\mathrm{q})=\mathrm{Q} \rightarrow \mathrm{Q}^{\prime}=>\mathrm{q}=$ route chosen for routing reorientation;

Because the analysis of alternate segments depends on the availability of resources, the definition of routing criteria in the protocol that establishes the agreed-upon communication rules is mandatory [21]. From this step, taking into account a search space that includes equipment adjacent to disrupted routers is the deductible possibility of continuing the transmission. In defining the search space of links to be chosen are also the fundamentals of dynamic communication from computer networks present due to the logical structure of routers.

It is necessary to understand from the measures defined for the identification of methods that will serve as a solution in reorienting transmissions is that the measurement indicts have dependencies between them. These dependencies are formulated to allow the choice of a route q. By using the relations (1) and (2) from [5] we have defined the choice process of route $q$ from the value pool $Q^{\prime}{ }_{1} \ldots . Q_{n}^{\prime}$, a pool that results from the evaluation of the selection space $\mathrm{Q}_{1} \ldots \mathrm{Q}_{\mathrm{n}}$. These relations impose the general performance conditions that the new communication route must meet once the functional process provides numerical runtime indicts that will later be used as a comparison template.

$$
\begin{aligned}
& \mathrm{N}=\mathrm{X} / \mathrm{R} \\
& \mathrm{U}=\mathrm{X} * \mathrm{~S}
\end{aligned}
$$

The conditions mentioned by the degree of throughput $\mathrm{X}$, together with the use of transmission points $\mathrm{U}$, allow the deduction of the selection space Q'. Given that 
the component transmission time must be met as an operating requirement, $\mathrm{q}$ is selected after defining the criteria from formula (3).

$$
\mathrm{q}=(\mathrm{X} * \mathrm{~S}) / \mathrm{T}
$$

$S$ represents the service time of the work tasks being multiplied by the throughput degree $\mathrm{X}$, a divisible product with extra workload time $\mathrm{T}$ that results in the identification of the point that can continue the transmission. The point of rerouting is primarily setup with the previous throughput parameters, having a node service time within the service limit and an optimal choice for transmitting data at $\mathrm{T}$ time intervals.

\section{Contributions and Related Work}

From the domain literature, where we can also give an example [22], two main branches can be distinguished as fundamental research, namely, the one focused on the probabilistic mathematics of Markov Chains and that of linear algorithms in Queueing Theory. In this study, we present the contributions through the prism of both theoretical formulations for analyzing information flows and we have developed the research stages according to the results found in the bibliography.

For a concise understanding of real-time communications, formula (4) simplistically describes real-time transmissions in terms of measured time, which is relevant in determining the communication time of a transmission protocol defined as having real-time actions. The notion of time in data communications is defined as global or individual per equipment and communication segment. This is how we write down the time $\mathrm{T}$ as the global time and $\mathrm{t}$ as the individual communication time per segment.

$$
\sum_{s=1}^{n} X_{s}=t_{s}-1=T
$$

In relation (4) $\mathrm{T}$ represents the overall communication time between the endpoints (data transmitters and receivers) of the real-time communication process and $t$ represents the communication time of each segment s. The summation of communication times forms the process in question, where $\mathrm{T}$ is presented as a result because the summed times of each segment with a communication performance $t$ must be limited and by reducing the amount of time with a quanta " 1 ", a real value is obtained that does not tend towards infinity in transmission. Noted with $\mathrm{X}_{\mathrm{s}}$ is the ability to pass a threshold directly related to global time $\mathrm{T}$ because only the generated packets that successfully meet the performance criteria are taken into account to calculate protocol-guided transmissions.

Real-time transmissions require an increased priority when communication media create them, these having as a requirement the fulfillment of two features with high significance. Firstly, real-time communications differ from typical Internet 
transmissions in that they require optimal correlation of transmission frames from segments with the general process of connecting the transmitter and receiver directly. This comes naturally as a deduction that synchronous algorithmic chaining is necessary for the fluidization of communications between endpoints [23]. Secondly, communications between endpoint entities require that protocol rules be considered concisely for session-level transmissions [24]. As a conclusion for the two mentioned functionalities, we mention that the communication rules guide all aspects of the operation of OSI session layer communications in realtime.

The created algorithm is designed to find an adjacent route that can meet the faulty/overloaded router's initial performance requirements on the network. For this action to happen, it implies an operation given by using the initial transmission times and the measurement of the adjacent segments' response time to define the route selection pool. Then, the transmission trip times to the destination are compared with the functional prior time values, and the next step in the algorithmic analysis is performed, namely the comparison of the optimal historical operation between the routers that are adjacent to the defect route. The granular metrics perform the comparison between routers operations and the adjacent segments pointed below. These are weighted to obtain uniform routing (without variations) for the persistent route that is to be further operated.

- $\quad \mathrm{N}_{\mathrm{a}}(\mathrm{t})=$ random process corresponding to the number of clients generated by the source (which arrive at the entrance to the service system);

- $\quad \mathrm{N}_{\mathrm{d}}(\mathrm{t})=$ random process corresponding to the number of customers leaving the service system;

- $\quad \mathrm{N}_{\mathrm{L}}(\mathrm{t})=$ random process corresponding to the number of customers rejected by the service system;

- $\quad \lambda_{\mathrm{ef}}=$ the effective rate of customers entering the system;

- $\quad \mathrm{N}_{\mathrm{q}}(\mathrm{t})=$ random process corresponding to the number of queued requests (en. queue), which are of a maximum length q;

- $\quad \mathrm{N}_{\mathrm{s}}(\mathrm{t})=$ random process corresponding to the number of in-service requests, in the "s" system servers;

The metrics that have the largest weight in the comparison process are $\mathrm{N}_{\mathrm{q}}(\mathrm{T})$ and $\mathrm{N}_{\mathrm{s}}(\mathrm{T})$, which show the size of the queues and the processing speed of data packets. The calculation in question significantly influences the selection probability of an adjacent network node because it considers the operations that are immediately necessary for continuing the persistent route. All this also implies the possibility that adjacent links can enter blocking states.

The routing reorientation process contains handshakes with neighboring routers [25], which before establishing an agreement they confirm if they can meet the requirements of real-time data transmission [17]. This requirement's 
accomplishment implies that routing is performed persistently on a new path chosen by equipment from the chosen equipment set to transmit the priority data.

In transmission media, time synchronization has a very basic role in highlighting the real-time process, which requires proper operation due to the proper management of networks. With time frames measured and embedded in the transmission process, the transmission media allows the observation of the transmission equipment's logical coordination, which in computer networks is more difficult to achieve due to complex functional dynamics. Through this possibility, the synchronization algorithm also allows dynamic management of network resources, which is a possibility that does not exist in alternative solutions that tolerate network faults.

An analysis of network devices' operation and the behavior of data flows was performed so that the relation between components that create real-time processes can be clear and obvious. We have defined relation (5) in order to achieve a uniform time-based classification for packets that are transmitted between adjacent routers and are partially reoriented in a session-level process.

$$
\frac{\lambda_{\mathrm{ef}} * \tau}{t} \cap \frac{N_{q}(t)+\mathrm{N}_{S}(t)}{\mathrm{O}^{\prime}} \rightarrow \mathrm{t}^{\prime}
$$

Expression (5) is the mathematical representation of the synchronization algorithm we propose. It is a cooperation of algorithmic embedded rules found in a protocol to reorient packets in routing pools by adjusting parameters related to the uniformity and beneficial correlation of the interactions which happen between network components.

In Fig. 3 we have illustrated the usefulness of relation (5) that has as a fundamental idea the functionality unification of the standard parameters that can meet real-time communication criteria for tolerating errors and malfunctions in transmitted sessions. We have used an ordering of functions in the light of Venn diagrams because each network and communication system that is transited requires its own operation and standardization through protocol rules. From this, we deduced that once a transmission medium is able to route data in real time it can contain sufficient communication resources through adjacent devices to satisfy the criteria of routing paths and distance vectors. These, in turn, can be completely differently when infrastructure subnets routers, and switches are disturbed. Data units make use of various parameters for rerouting packets in case intermediate defects occur [26] and for the formulas illustrated in Fig. 3 to be clearly transposed in the real world of computer networks, we mention that time $t$ ' from relation (5) must be understood as a component of the set given by the value range $Q$ and service rate $S$.

In the following paragraphs, we will detail the intersection formed in relation (5) for running a communication protocol that we have logically defined to perform the synchronization algorithm proposed as a solution. 
Relation (5) contains the following performance metrics:

- $\quad \lambda_{\mathrm{ef}}=$ the effective rate of requests entering the system;

- $\tau=$ random variable that represents the actual service time;

- $\quad \mathrm{N}_{\mathrm{q}}(\mathrm{t})=$ random process corresponding to the number of range queue requests of maximum length q;

- $\quad \mathrm{N}_{\mathrm{s}}(\mathrm{t})=$ random process corresponding to the number of requests that are in service, in the s system servers;

- $\quad t=$ the communication time between two network hosts and/or the endpoints that form the real-time communication process;

- $\quad \mathrm{Q}^{\prime}=$ segments adjacent to the affected one, which met the necessary parameters to be able to take over real-time data communications;

The solution is described mathematically in relation (5) and it represents the details of the brief presentation from the introduction. The approach is complex. It contains a multitude of network mechanisms, functions, parameters, and metrics that operate interconnected computer networks only up to the OSI model's network level [28]. In order for the proposed protocol, the synchronization algorithm, and the interaction mechanism to be usable in networking environments, the following mentions are deduced:

- The performance metrics that we have studied as methods to impose the selfadaptation of communication parameters need to be included in the protocol so that it can gradually analyze parameters and performance limits of routing devices;

- The self-adaptation of the communication process to the actual necessary rerouting feature must be carried out within the negotiated time limits, primarily to comply with end-to-end agreements and secondly to be applicable in terms of times $\tau$ and $\mathrm{N}_{\mathrm{q}}(\mathrm{T})$;

- The functions defined by the basic protocols of computer networks must be used in the decision making process for mitigating the in-cause disturbances, these being based on the specifications of mechanisms that use performance indices which are implied in reorienting tcp datagrams;

The three points mentioned above are a highlight of computational interactions. In essence, they aim to formalize for the real-time transmission process what the communication protocol needs to do to mitigate possible disruptions of damaged transmission [29]. 


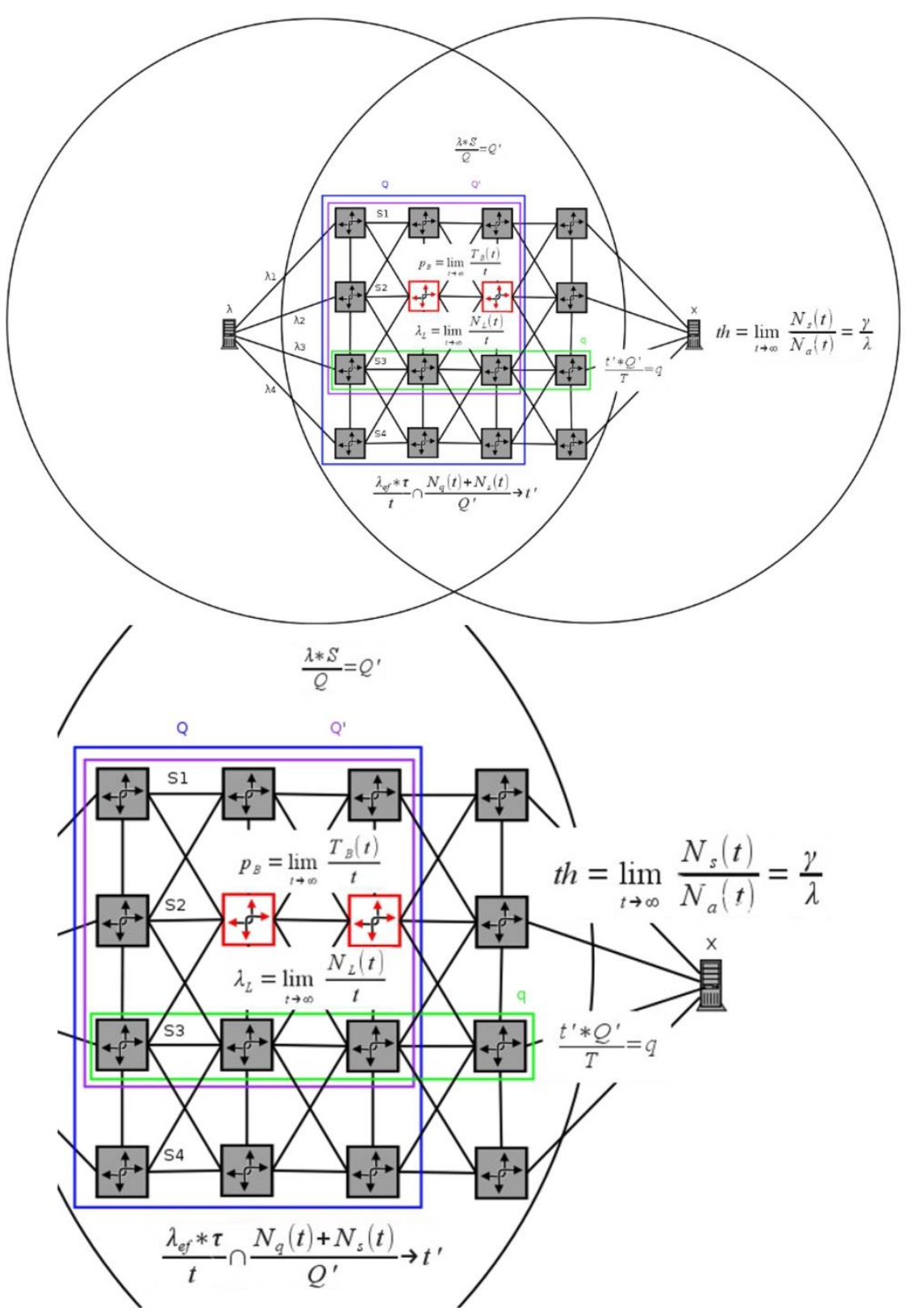

Figure 3

Visualization of the performance analysis of the algorithm [27] 
Because interaction has a well-defined role (as a concept) in categorizing actions from networks, we considered that each event (action and reaction) could be integrated with a beneficial purpose in analyzing of traffic problems and in particular in the analysis of stability and trust.

The lead author of the OSI model specified for ISO a layered organization of functional dependencies that aims to determine the technical requirements of transmissions performed between various network points and with different services. These requirements mainly need a correct operation of each previous layer to meet the performance requirements of the level at which the analyzed service is. If layer 6 presentation requires real-time data, all infrastructure (physical data link and networks) and logical (session and transport levels) criteria must be met according to the presentation layer service specifications. These can be generic (e.g. the bit length of an IP packet), but routing in itself is created as a process by the need to provide the solicited resources of the application/program in question.

For the session layer of the OSI model, there are many mechanisms that make use of several protocols at once [30]. These make general use of tcp level variance agreements to provide for session routing processes the performances that routing devices have available. For example, the 3-way handshake also requests an acknowledgement for a transmitted data packet in order to assure the sender of the data packets that they have been received. Another example is the agreement of the SIP protocol with 8 directions, in which every step of the initiation in question requires a confirmation, an acknowledgment used also for maintaining the established data flow. This maintenance is necessary for the dimensioning and structuring of fragmented and transmitted data packets [31]. Examples of technologies and mechanisms that require multiple correct operations in real-time are VPN, IPsec, RTP, and in general any connection-oriented transmission to which quality requirements apply.

Since overlapping OSI requirements have a way to gradually edify each technology that will be part of a transmission with specific attributes, the anomalies that may occur through disfunctions of protocol agreements are those that indicate the significance of parameters in certain contexts. There are cases where the parameters are generic, but these parameters are the most important for guaranteed quality of service. For example, the arrival and processing times of data packets indicate the speed of execution for processing centers [32], which is a current research topic [33]. To emphasize these things, we wanted to describe as accurately as possible from a logical and mathematical point of view the operation of sessions in real-time. This description was presented throughout this article by explaining and representing the functionality of real-time session generation processes and their maintenance in an active state with an optimal runtime. 


\section{Conclusions}

The ideas outlined in this paper involve a theoretical comprehensive consideration on the technological ensemble through which persistent routing is performed, allowing the attribution of the reliable communication characteristic. Ensuring a real-time communication process to be as part of reliability and dependability values is given by the analysis on routing resources through which disruptive effects are mitigated, and routing is maintained as functional after the usage of routes that were selected in performance terms.

Being a solution that must be adapted to the field of communication networks and in particular to the international norms applied at a global level, the presentation of the concept of interaction requires the analysis and troubleshooting of OSI session-level malfunctions through the synchronization algorithm. It is operated by performance metrics that are in accordance with the protocol rules for the formation of transmissions. The protocol ruleset was created and analyzed both by technical comparisons and by concrete analyzes of unitary equipment and networking environments. They are described in the study as a necessary criterion in fulfilling fault tolerance of real-time failures.

Any communication protocol makes use of functional criteria for both guaranteed transmissions in terms of quality, as well as for communications based on retransmission algorithmic efforts. In this way, the operating criteria were established by using routing indicatives that can be optimized with the desired parametric specification.

\section{References}

[1] A. Burns, A. Wellings, Real-Time Systems and Programming Languages, Second Edition, Addison Wesley, 1997

[2] A. T. Campbell, H. G. de Meer, M. E. Kounavis, K. Miki, J. B. Vicente, D. Villela, A Survey of Programmable Networks, ACM SIGCOMM, Vol. 29, Issue 2, pp. 7-23, April 1999

[3] AS Tanenbaum, Computer Networks, $4^{\text {th }}$ Edition, Byblos, 2004

[4] AS Tanenbaum, Modern Operating Systems, Second Edition, Byblos, 2004

[5] E. D. Lazowska, J. Zahorjan, G. S. Graham, K. C. Sevcik, Quantitative System Performance: Computer System Analysis Using Queueing Network Models, Prentice Hall, 1984

[6] M. As. Arfeen, K. Pawlikowski, A. Willig, D. McNickle, Internet traffic modeling: from superposition to scaling, IET Netw., Vol. 3, Iss. 1, 2014

[7] F. R. Barbosa, I. B. Martins, Y. Martins, E. Moschim, Comparative Study of Latency and Throughput in DPS / DBS Metro-Access Networks, $16^{\text {th }}$ International Telecommunications Network Strategy and Planning Symposium (Networks), Funchal, Portugal, DOI: 10.1109/NETWKS.2014.6959222, 2014 
[8] D. Darshana, M. Chatterjee, K. Kwiat, Delay Based Routing for Real-time Traffic in Ad Hoc Networks, The 2010 International Conference on Computer Engineering \& Systems, Cairo, Egypt, DOI: 10.1109/ICCES.2010.5674901, ISBN: 978-1-4244-7042-6, 2010

[9] T. Hirai, S. Ohzahata, K. Kawashima, A TCP Congestion Control Method for Real-Time Communication Based on Channel Occupancy of a Wireless LAN, $16^{\text {th }}$ Asia-Pacific Conference on Communications (APCC), Auckland, New Zealand, ISBN: 978-1-4244-8129-3, DOI: 10.1109/APCC.2010.5679755, 2010

[10] R. Baumann, S. Heimlicher, M. Strasser, A. Weibel, A Survey on Routing Metrics, Computer Engineering and Networks Laboratory ETH - Zentrum, Switzerland, February 10, 2007

[11] C. Boler, S. Yenduri, Resilient Multi Sink Networks Using Simplistic Hop based Routing, $11^{\text {th }}$ International Conference on Information Technology: New Generations, Las Vegas, USA, ISBN: 978-1-4799-3188-0, DOI: 10.1109/ITNG.2014.11, 2014

[12] M. Csernai, A. Gulyas, G. Retvari, Z. Heszberger, A. Csaszar, The Skeleton of the Internet, IEEE Global Telecommunications Conference GLOBECOM, Miami, USA, ISBN: 978-1-4244-5638-3, DOI: 10.1109/GLOCOM.2010.5684319, 2010

[13] Y. Cui, P. Wu, M. Xu, J. Wu, YL Lee, A. Durand, C. Metz, 4over6: Network Layer Virtualization for Ipv4-IPv6 Coexistence, IEEE Network, Issue September-October 2012

[14] WJ Dally, B. Towles, Route Packets, Not Wires: On-Chip Interconnection Networks, Computer Systems Laboratory Stanford University, Stanford, Proceedings of the $38^{\text {th }}$ Design Automation Conference (IEEE Cat. No.01CH37232), Las Vegas, USA, ISBN: 1-58113-297-2, DOI: 10.1109/DAC.2001.156225, 2001

[15] D. Duolikun, Makoto. Takizawa, Communication Protocols in Layered Groups with Heterogeneous Clocks, Seventh International Conference on Broadband, Wireless Computing, Communication and Applications, Victoria, Canada, ISBN: 978-1-4673-2972-9, DOI: 10.1109/BWCCA.2012.100, 2012

[16] A. Thekkilakattil, R. Dobrin, S. Punnekkat, Mixed Criticality Scheduling andn Fault-Tolerant Distributed Real-Time Systems, International Conference on Embedded Systems (ICES), Coimbatore, India, ISBN: 9781-4799-5026-3, DOI: 10.1109/EmbeddedSys.2014.6953097, 2014

[17] O. Fatmi, D. Pan, Distributed Multipath Routing for Data Center Networks based on Stochastic Traffic Modeling, Proceedings of the $11^{\text {th }}$ IEEE International Conference on Networking, Sensing and Control, Miami, USA, ISBN: 978-1-4799-3106-4, DOI: 10.1109/ICNSC.2014.6819683, 2014 
[18] G. V. Bochmann, C. A. Sunshine, Formal Methods in Communication Protocol Design, IEEE Transactions on Communications, Vol. Com-28, No. 4, April 1980

[19] V. Ciancaglini, L. Liquori, GN Hoang, Towards a common architecture to interconnect heterogeneous overlay networks, IEEE $17^{\text {th }}$ International Conference on Parallel and Distributed Systems, Tainan, Taiwan, ISBN: 978-0-7695-4576-9, DOI: 10.1109/ICPADS.2011.139, 2011

[20] T. Condeixa, S. Sargento, Centralized, Distributed or Replicated IP Mobility, IEEE Communications Letters, Vol. 18, No. 2, February 2014

[21] D. Eastlake, A. Banerjee, D. Dutt, R. Perlman, A. Ghanwani, Transparent Interconnection of Lots of Links (TRILL) Use of IS-IS, Internet Engineering Task Force (IETF), Request for Comments: 6326, Category: Standards Track, ISSN: 2070-1721, July 2011

[22] A. M. Farhangi, A. J. Al-Khalili, D. Al-Khalili, Pattern-Driven Clock Tree Routing with Via Minimization, IEEE Computer Society Annual Symposium on VLSI, Lixouri, Greece, ISBN: 978-1-4244-7320-5, DOI: 10.1109/ISVLSI.2010.82, 2010

[23] N. Feamster, H. Balakrishnan, J. Rexford, A. Shaikh, J. van der Merwe, The Case for Separating Routing from Routers, SIGCOMM'04 Workshops, Aug. 30-Sept. 3, 2004, Portland, Oregon, USA

[24] Y. Fouquet, D. Nace, M. Pioro, M. Poss, M. Zotkiewics, Flow adjustment methods for survivable networks, $16^{\text {th }}$ International Telecommunications Network Strategy and Planning Symposium (Networks), Funchal, Portugal, ISBN: 978-1-4799-6515-1, DOI: 10.1109/NETWKS.2014.6959261, 2014

[25] L. Gao, On Inferring Autonomous System Relations In The Internet, IEEE / ACM Transactions On Networking, Vol. 9, No. 6, December 2001

[26] J. Wu, T. Fang, C. Chen, C. Zhang, A Hierarchical Quadtree-Based Link State Routing Scheme for Routing Compression Table, International Conference on Networking and Network Applications (NaNA), Hakodate, Japan, ISBN: 978-1-4673-9803-9, DOI: 10.1109/NaNA.2016.38, 2016

[27] S. N. Orzen, M. Stratulat, S. Babii, C. Cosovan, Markov Chains State Transitions for Reliable Persistent Routing, 2016 IEEE $20^{\text {th }}$ Jubilee International Conference on Intelligent Engineering Systems (INES), Budapest, Hungary, DOI: 10.1109/INES.2016.7555101, ISBN:978-1-50901216-9, 2016

[28] S. N. Orzen, S. Babii, Network Events in the Dynamic Selection of RealTime Session Fault Tolerant Routes, 2017 IEEE $21^{\text {st }}$ International Conference on Intelligent Engineering Systems (INES), Larnaca, Cyprus, DOI: 10.1109/INES.2017.8118574, ISBN:978-1-4799-7678-2, 2018 
[29] S. N. Orzen, S. Babii, Data Packet Header Actions in Fault Tolerance of Persistent Routing Sessions, 2017 IEEE 15 ${ }^{\text {th }}$ International Symposium on Intelligent Systems and Informatics (SISY), Subotica, Serbia, DOI: 10.1109/SISY.2017.8080551, ISBN:978-1-5386-3855-2, 2017

[30] J. Kuo, C. Shih, Y. Chen, A Cross-Layer Design for P2P Live Streaming with Graceful Handover in Mobile IP Network, $13^{\text {th }}$ International Conference on ITS Telecommunications (ITST), Tampere, Finland, ISBN: 978-1-4799-0846-2, DOI: 10.1109/ITST.2013.6685588, 2013

[31] G. G. Patrushev, V. G. Drozdova, Routing Efficiency Evaluation with SDN Solutions Integration in the Data Network, International Conference on Micro / Nanotechnologies and Electron Devices 2017

[32] S. N. Orzen, M. Stratulat, Performance Queues for Fault Tolerant Sessions, 2018 International Symposium on Electronics and Telecommunications (ISETC), Timisoara, Romania, DOI: 10.1109/ISETC.2018.8583983, ISBN: 978-1-5386-5925-0, 2018

[33] V. E. Gvozdev, L. R. Chernyakhovskaya, D. V. Blinova, D. R. Akhmetova, Analysis of the GRID's Basic Topological Structure Reliability, Acta Polytehnica Hungarica, Vol. 17, No. 8, 2020 